\title{
Solitons, Shock Waves, Conservation Laws and Bifurcation Analysis of Boussinesq Equation with Power Law Nonlinearity and Dual Dispersion
}

\author{
Anjan Biswas ${ }^{1,2, *}$, Ming Song ${ }^{3}$, Houria Triki ${ }^{4}$, Abdul H. Kara ${ }^{5}$, Bouthina S. Ahmed ${ }^{6}$, Andre Strong ${ }^{1}$ and Amadou \\ Hama $^{1}$ \\ ${ }^{1}$ Department of Mathematical Sciences, Delaware State University, Dover, DE 19901-2277, USA \\ 2 Department of Mathematics, Faculty of Science, King Abdulaziz University, Jeddah, Saudi Arabia \\ ${ }^{3}$ Department of Mathematics, Yuxi Normal University, Yuxi-653100, China \\ ${ }^{4}$ Radiation Physics Laboratory, Department of Physics, Faculty of Sciences, Badji Mokhtar University, P. O. Box 12, 23000 Annaba, \\ Algeria \\ ${ }^{5}$ School of Mathematics, Centre for Differential Equations, Continuum Mechanics and Applications, University of the Witwatersrand \\ , Johannesburg, Wits 2050, South Africa \\ ${ }^{6}$ Department of Mathematics, College of Girls, Ain Shams University, Cairo, Egypt
}

Received: 5 May. 2013, Revised: 9 Sep. 2013, Accepted: 10 Sep. 2013

Published online: 1 May. 2014

\begin{abstract}
This paper obtains the soliton solutions to the Boussinesq equation with the effect of surface tension being taken into account. The power law nonlinearity is considered. Three integration tools are adopted in order to extract the soliton solutions. They are the traveling wave hypothesis, ansatz method and the semi-inverse variational principle. Finally, the Lie symmetry approach is adopted to extract the conservation laws of this equation.
\end{abstract}

Keywords: Boussinesq equation, soliton solutions, variational principle

\section{Introduction}

The dynamics of shallow water waves along ocean and sea shores are modeled by several forms of nonlinear evolution equations (NLEEs) $[1,2,3,4,5,6,7,8,9,10,11$, $12,13,14,15,16,17,18,19,20,21,22]$. A few of the well-known models are Korteweg-de Vries (KdV) equation, Peregrine equation, Benjamin-Bona-Mahoney equation. On the other had two-layered shallow water waves are typically modeled by the Bona-Chen equation, Gear-Grimshaw model, coupled KdV equation and many such coupled equations. This paper will focus on one such NLEE that models shallow water wave dynamics along with the effect of surface tension. This is the Boussinesq equation (BE). The effect of surface tension will introduce a second dispersion term and hence the name dual-dispersion. For a generalized setting, BE will be studied with power law nonlinearity.
The main focus of this paper is going to be the integrability aspect of this model. While there are several approaches to the integrability aspect of NLEEs, one needs to exercise caution while administering the integration process. Some of the commonly applied integration architectures are the exp-function approach, $G^{\prime} / G$-expansion method, simplest equation method, Lie symmetry approach, differential transformation technique, variational iteration method, Adomian decomposition method and quite a few others. However, this paper will apply the classic travel wave hypothesis where a wave of permanent form will be revealed. The ansatz approach will be utilized to extract the solitary wave and singular soliton solutions to the BE. The semi-inverse variational method will also be applied as a third tool to solve the BE. Subsequently, the multiplier method using Lie symmetry approach will lead to the conservation laws of the equation. Finally, the bifurcation analysis will for the BE will be seen.

\footnotetext{
*Corresponding author e-mail: biswas.anjan@gmail.com
} 


\section{TRAVELING WAVE SOLUTION}

The dimensionless form of the BE with power law nonlinearity and dual-dispersion is given by [18]

$q_{t t}-k^{2} q_{x x}+a\left(q^{2 n}\right)_{x x}+b_{1} q_{x x x x}+b_{2} q_{x x t t}=0$

In (1), the dependent variable $q(x, t)$ is the wave profile while $x$ and $t$ are the spatial and temporal variables respectively. Also, $k, a$ and $b_{j}$ for $j=1,2$ are real-valued constants. The first two terms constitute the wave operator, where the first term is the linear evolution term. The coefficient of $a$ represents the nonlinear term where the parameter $n$ is the power law nonlinearity parameters. Typically, the case $n=1$ is studied in the literature of Ocean Dynamics. However, this paper will leave $n$ as an arbitrary power law parameter in order to give BE a generalized flavor. Then, the coefficients of $b_{j}$ are the two dispersion terms. The coefficient of $b_{1}$ is the regular dispersion, while the second dispersion term that is given by the coefficient of $b_{2}$ accounts for the surface tension [18]. The soliton solutions to (1) will be the only issue of this paper that can be formulated only when a delicate balance between dispersion and nonlinearity is in place.

In order to seek traveling wave solution to (1), the hypothesis [8]

$q(x, t)=g(x-v t)$

where $g(s)$ represents the wave of permanent form with

$s=x-v t$

and $v$ represents the velocity of the wave. Substituting the hypothesis (2) into (1) leads to the ordinary differential equation (ODE) that is given by

$\left(v^{2}-k^{2}\right) g^{\prime \prime}+a\left(g^{2 n}\right)^{\prime \prime}+\left(b_{1}+b_{2} v^{2}\right) g^{\prime \prime \prime \prime}=0$

Integrating (4) twice, leads to

$\left(v^{2}-k^{2}\right) g+a g^{2 n}+\left(b_{1}+b_{2} v^{2}\right) g^{\prime \prime}=0$

after choosing the integration constant to be zero, since the search is for a soliton solution. Multiplying both sides of (5) by $g^{\prime}$ and integrating one more time, again after taking the integration constant to be zero implies

$\frac{d g}{d s}=\frac{g}{\sqrt{b_{1}+b_{2} v^{2}}} \sqrt{k^{2}-v^{2}-\frac{2 a g^{2 n-1}}{2 n+1}}$

After separating variables and integrating yields

$g(s)=g(x-v t)=q(x, t)=A \operatorname{sech}^{\frac{2}{2 n-1}}[B(x-v t)]$

where the amplitude $A$ of the soliton is

$A=\left[\frac{(2 n+1)\left(k^{2}-v^{2}\right)}{2 a}\right]^{\frac{1}{2 n-1}}$

while the inverse width of the soliton is

$B=\frac{2 n-1}{2} \sqrt{\frac{k^{2}-v^{2}}{b_{1}+b_{2} v^{2}}}$
The soliton width given by (9) provokes a constraint condition that is given by

$$
\left(k^{2}-v^{2}\right)\left(b_{1}+b_{2} v^{2}\right)>0
$$

Upon setting $b_{2}=0$ in (1) and (9) the equation and consequently the corresponding result collapses to the regular BE with power law nonlinearity that has been studied earlier [8].

\section{ANSATZ METHOD}

This section will focus on another approach to integrating the BE. This is the ansatz method. Using a judicious hypothesis, a soliton solution will be obtained to the BE. While the traveling wave hypothesis revealed a solitary wave solution, this approach, however, is unable to obtain singular or topological soliton solution to this equation. The appealing ansatz method will therefore lead to a better picture for the BE. The hypothesis will be based on the type of soliton solution that is being sought for. Therefore the study of ansatz approach will be split into the following three subsections.

\subsection{SOLITARY WAVES}

In order to solve equation (1) for the solitary waves, the starting hypothesis is given by [15]

$q(x, t)=A \operatorname{sech}^{p} \tau$

Here in (11), $A$ and $B$ represent the amplitude and the inverse width of the soliton. The definition of $\tau$ is given by

$\tau=B(x-v t)$

Substituting this hypothesis into (1) and simplifying leads to

$$
\begin{aligned}
& p A\left\{v^{2}-k^{2}+\left(b_{1}+b_{2} v^{2}\right) p^{2} B^{2}\right\} \operatorname{sech}^{p} \tau \\
- & (p+1) A\left(v^{2}-k^{2}\right) A B \operatorname{sech}^{p+2} \\
+ & \left\{p^{2}+(p+2)^{2}\right\}\left(b_{1}+b_{2} v^{2}\right) A B^{3} \operatorname{sech}^{p+2} \tau \\
+ & A B^{2}(p+1)(p+2)(p+3)\left(b_{1}+b_{2} v^{2}\right) \operatorname{sech}^{p+4} \tau \\
+ & 4 a n^{2} p A^{2 n} \operatorname{sech}^{2 n p} \tau \\
- & 2 a n(2 n p+1) A^{2 n} \operatorname{sech}^{2 n p+2} \tau=0
\end{aligned}
$$

From (13) equating the exponents $2 n p$ and $p+2$ leads to

$2 n p=p+2$

so that

$p=\frac{2}{2 n-1}$

One important observation is that the same value of the unknown exponent $p$ is obtained upon equating the exponents $2 n p+2$ and $p+4$. Now, from (13), setting the 
coefficients of the linearly independent functions $\operatorname{sech}^{p+j} \tau$ to zero, for $j=0,2,4$, leads to (9) as well as

$A=\left[\frac{(2 n+1)\left\{\left(v^{2}-k^{2}\right)(2 n-1)^{2}+\eta\right\}}{8 n^{2}(2 n-1)^{2} a}\right]^{\frac{1}{2 n-1}}$

where $\eta=4\left(4 n^{2}+1\right)\left(b_{1}+b_{2} v^{2}\right) B^{2}$, and

$A=\left[\frac{2(2 n+1)\left(b_{1}+b_{2} v^{2}\right) B^{2}}{(2 n-1)^{2} a}\right]^{\frac{1}{2 n-1}}$

Next, upon equating the two values of the amplitude $A$ from (16) and (17) leads to (9). Thus the system of relations from the coefficients of the linearly independent functions, leads to a closure. Hence, the 1-soliton solution to the $\mathrm{BE}$ is given by (7) with the amplitude $(A)$ given by (16) or (17) while the width $(B)$ is in (9) along with the constraint condition that is given by (10).

The following figure shows the profile of a solitary wave for $a=1, b_{1}=1, b_{2}=1, k=1$ and $n=1$.

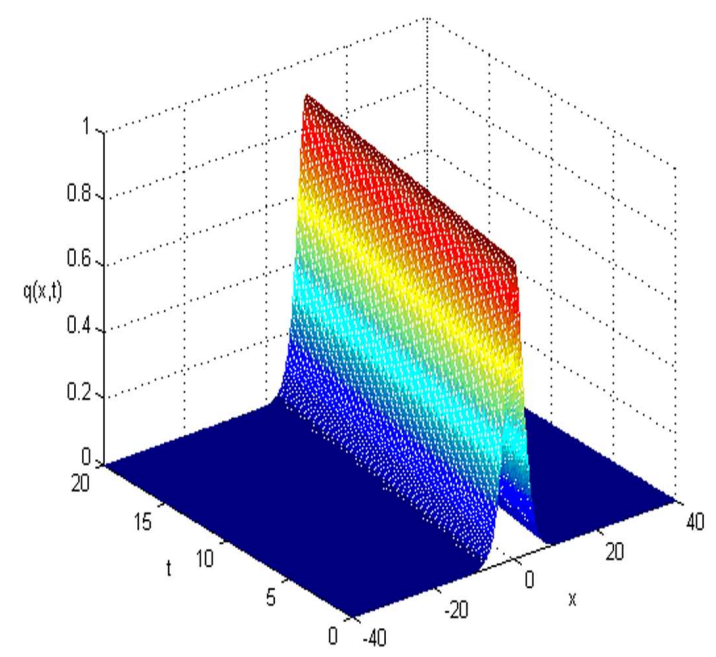

Figure 1: Solitary wave $q(x, t)$ with $n=1, a=1, b 1=1, b 2=1, k=1, b=0.5,-40<x<40,0<t<20$

\subsection{SHOCK WAVES}

Shock waves, that are also known as topological solitons or kinks are studied in the context of fluid dynamics. Thus, for topological soliton solution, the starting hypothesis is taken to be [13]

$$
q(x, t)=A \tanh ^{p} \tau
$$

In this case, the parameters $A$ and $B$ are free parameters. Therefore substituting this hypothesis into (1) leads to

$$
\begin{aligned}
& \left(v^{2}-k^{2}\right) A B\left\{(p-1) \tanh ^{p-2} \tau-2 p \tanh ^{p} \tau\right\} \\
+ & (p+1) \tanh ^{p+2} \tau+2 a n A^{2 n} B(2 p n-1) \tanh ^{2 p n-2} \tau \\
- & 2 a n A^{2 n} B\left\{4 p n \tanh ^{2 p n} \tau-(2 p n+1) \tanh ^{2 p n+2} \tau\right\} \\
+ & \left(b_{1}+b_{2} v^{2}\right) A B^{3}\left[(p-1)(p-2)(p-3) \tanh ^{p-4} \tau\right. \\
+ & (p+1)(p+2)(p+3) \tanh ^{p+4} \tau \\
- & 2(p-1)\left\{p^{2}+(p-2)^{2}\right\} \tanh ^{p-2} \tau \\
- & 2(p+1)\left\{p^{2}+(p+2)^{2}\right\} \tanh ^{p+2} \tau \\
+ & 4 p^{3} \tanh ^{p}+(p-1)^{2}(p-2) \tanh ^{p} \\
+ & \left.(p+1)^{2}(p+2) \tanh ^{p} \tau\right]=0
\end{aligned}
$$

In this case from coefficient of the stand-alone linearly independent function is $\tanh ^{p-4} \tau$ and the coefficient of the linearly independent function $\tanh ^{p-2} \tau$ leads to

$$
p=1
$$

Then again, equating the exponents $2 n p$ and $p+2$ as in the case of solitary waves in the previous section leads to (15) which therefore implies

$$
n=\frac{3}{2}
$$

Hence the power law BE that supports topological soliton solution is given by

$q_{t t}-k^{2} q_{x x}+a\left(q^{3}\right)_{x x}+b_{1} q_{x x x x}+b_{2} q_{x x t t}=0$

Now, from (17) setting the coefficients of the linearly independent functions $\tanh ^{p+j} \tau$, for $j=0,2,4$, to zero leads to

$$
\begin{gathered}
B=\frac{A}{2} \sqrt{-\frac{a}{b_{1}+b_{2} v^{2}}} \\
3 a A^{2}+8\left(b_{1}+b_{2} v^{2}\right) B^{2}=v^{2}-k^{2}
\end{gathered}
$$

and

$$
9 a A^{2}+20\left(b_{1}+b_{2} v^{2}\right) B^{2}=v^{2}-k^{2}
$$

From (24) and (25) subtracting one from the other, the same relation between the free parameters as in (23) is revealed. This free parameters' relation kicks in a constraint condition given by

$$
a\left(b_{1}+b_{2} v^{2}\right)<0
$$

in order for the shock waves to exist.

So, finally, the shock solution to the BE with power law nonlinearity and dual-dispersion exists only when the power law nonlinearity collapses to cubic nonlinearity. This is being observed for the very first time in this paper. In this case, the shock wave solution is given by

$$
q(x, t)=A \tanh [B(x-v t)]
$$


where the relation between the free parameters is depicted in (21). Additionally, the constraint relation given by (24) must hold in order for these kinks to exist.

The following figure shows the profile of a shock wave for $p=1, a=1, b_{1}=b_{2}=1, k=1, A=1$.

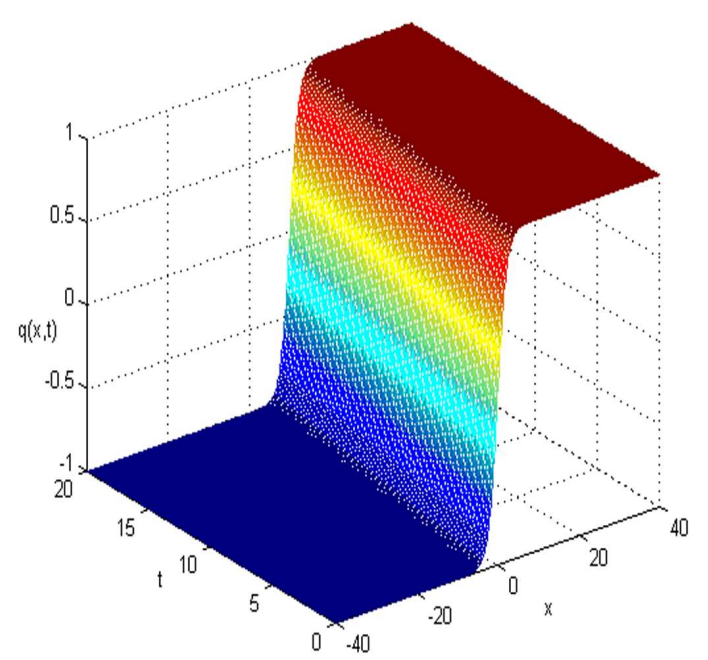

Figure 2. Shock wave $q(x, t)$ with $p=1, a=-1, b 1=1, b 2=1, k=1, v=0.5,-4<x<40,0<t<20$.

\subsection{SINGULAR SOLITON}

Singular solitons are the third kind of solitons that provide a possible analytical explanation to the formation of rogue waves. In order to obtain the singular soliton solution, the starting hypothesis is given by

$q(x, t)=A \operatorname{csch}^{p} \tau$

where the definition of $\tau$ stays the same as in (12). However, the parameters $A$ and $B$ is this case are free parameters. Substituting this hypothesis into (1) and simplifying leads to

$$
\begin{aligned}
& p A\left\{v^{2}-k^{2}+\left(b_{1}+b_{2} v^{2}\right) p^{2} B^{2}\right\} \operatorname{csch}^{p} \tau \\
- & (p+1) A\left(v^{2}-k^{2}\right) \operatorname{csch}^{p+2} \tau \\
+ & (p+1) A\left[\left\{p^{2}+(p+2)^{2}\right\}\left(b_{1}+b_{2} v^{2}\right) B^{2}\right] \operatorname{csch}^{p+2} \tau \\
+ & A B^{2}(p+1)(p+2)(p+3)\left(b_{1}+b_{2} v^{2}\right) \operatorname{csch}^{p+4} \tau \\
+ & 4 a n^{2} p A^{2 n} \operatorname{csch}^{2 n p} \tau \\
- & 2 a n(2 n p+1) A^{2 n} \operatorname{csch}^{2 n p+2} \tau=0
\end{aligned}
$$

The balancing principle again gives (15). Similarly, as in the case of solitary waves, the coefficients of the linearly independent functions $\operatorname{csch}^{p+j} \tau$ for $j=0,2,4$ leads to the relations (9) as well as

$A=\left[-\frac{(2 n+1)\left\{\left(v^{2}-k^{2}\right)(2 n-1)^{2}+\theta\right\}}{8 n^{2}(2 n-1)^{2} a}\right]^{\frac{1}{2 n-1}}$

where $\theta=4\left(4 n^{2}+1\right)\left(b_{1}+b_{2} v^{2}\right) B^{2}$, and

$A=\left[-\frac{2(2 n+1)\left(b_{1}+b_{2} v^{2}\right) B^{2}}{(2 n-1)^{2} a}\right]^{\frac{1}{2 n-1}}$.
Again, upon equating the two values of the parameter $A$ from (30) and (31), leads to the same value of $B$ as in (9). Thus, the singular 1-soliton solution to (1) is

$q(x, t)=A \operatorname{csch}^{\frac{2}{2 n-1}}[B(x-v t)]$

where the free paremter $A$ is given by (30) or (31) while the parameter $B$ is in (9) with the constraint condition (10).

\section{OBSERVATION}

This section will discuss an interesting connection between the solitary wave solution and the singular soliton solution. For the special case when $n=1$, equation (1) modifies to

$q_{t t}-k^{2} q_{x x}+a\left(q^{2}\right)_{x x}+b_{1} q_{x x x x}+b_{2} q_{x x t t}=0$

whose solitary wave solution can be written as

$q(x, t)=A \operatorname{sech}^{2}\left[B(x-v t)-x_{0}\right]$,

where $x_{0}$ is the center position of the soliton, and the singular soliton solution can be written as

$q(x, t)=A \operatorname{csch}^{2}[B(x-v t)]$

after the respective parameters $A$ and $B$ are modified with respect to the specific value $n=1$. It can be easily observed that the singular soliton solution (34) can be easily re-casted into (35) by implementing the transformation

$e^{2 x_{0}}=-1$.

Therefore, the singular solitons can be recovered from solitary waves using this exponential transformation with regards to the center position.

\section{SEMI-INVERSE VARIATIONAL PRINCIPLE}

This semi-inverse variational principle (SVP) is the third alternative scheme to solve the governing equation. One must note that the special case of the governing equation was solved earlier by the aid of SVP [6]. The starting hypothesis to solve (1) is the traveling wave hypothesis that is given by (2). Next, multiplying both sides of (5) by $g^{\prime}$ and integrating both sides with respect to s, gives

$$
\left(v^{2}-k^{2}\right) g^{2}+\left(b_{1}+b_{2} v^{2}\right)\left(g^{\prime}\right)^{2}+\frac{2 a}{2 n+1} g^{2 n+1}=K
$$

where $K$ is an integration constant. The stationary integral $J$ is then defined as [6]

$J=\int_{-\infty}^{\infty} K d s$

which is therefore

$J=\int_{-\infty}^{\infty}\left[\left(v^{2}-k^{2}\right) g^{2}+\left(b_{1}+b_{2} v^{2}\right)\left(g^{\prime}\right)^{2}+\vartheta\right] d s$ 
where $\vartheta=\frac{2 a}{2 n+1} g^{2 n+1}$. The 1-soliton solution assumption to (1) is [6]

$g(s)=A \operatorname{sech}^{\frac{2}{2 n-1}}(B s)$.

Inserting (40) into (39) and carrying out the integrations yield

$$
\begin{aligned}
J & =\left\{\left(v^{2}-k^{2}\right) \frac{A^{2}}{B}+\frac{4\left(b_{1}+b_{2} v^{2}\right) A^{2} B}{(2 n-1)(2 n+3)}\right. \\
& \left.+\frac{8 a}{(2 n+1)(2 n+3)} \frac{A^{2 n+1}}{B}\right\} \frac{\Gamma\left(\frac{2}{2 n-1}\right) \Gamma\left(\frac{1}{2}\right)}{\Gamma\left(\frac{2}{2 n-1}+\frac{1}{2}\right)}
\end{aligned}
$$

after integration, where $\Gamma(x)$ is the Euler's gamma function. The variational principle states that the parameters $A$ and $B$ are so chosen that [6]

$\frac{\partial J}{\partial A}=0$

and

$\frac{\partial J}{\partial B}=0$.

Thus from (41), equations (42) and (43), after simplification, respectively are

$v^{2}-k^{2}+\frac{4\left(b_{1}+b_{2} v^{2}\right) B^{2}}{(2 n-1)(2 n+3)}+\frac{4 a A^{2 n-1}}{2 n+3}=0$

and

$v^{2}-k^{2}-\frac{4\left(b_{1}+b_{2} v^{2}\right) B^{2}}{(2 n-1)(2 n+3)}+\frac{8 a A^{2 n-1}}{(2 n+1)(2 n+3)}=0$

Equations (44) and (45), after uncoupling, gives the soliton amplitude $(A)$ as given by $(8)$ and the width $(B)$ as

$B=\frac{2 n-1}{2} \sqrt{\frac{v^{2}-k^{2}}{b_{1}+b_{2} v^{2}}}$

which induces the constraint condition

$\left(b_{1}+b_{2} v^{2}\right)\left(v^{2}-k^{2}\right)>0$.

Also, equations (8) and (46) produces the amplitude-width relationship as

$B=(2 n-1)\left[-\frac{a A^{2 n-1}}{(2 n+1)\left(b_{1}+b_{2} v^{2}\right)}\right]^{\frac{1}{2}}$

which poses the condition

$a\left(b_{1}+b_{2} v^{2}\right)<0$

in order for the solitons to exist. Once again, it needs to be noted that the results of this section is a generalized version of the results that was reported in 2013 [6].

\section{CONSERVATION LAWS}

In order to determine conserved densities and fluxes, we resort to the invariance and multiplier approach based on the well known result that the Euler-Lagrange operator annihilates a total divergence (see [10]). Firstly, if $\left(T^{t}, T^{x}\right)$ is a conserved vector corresponding to a conservation law, then

$$
D_{t} T^{t}+D_{x} T^{x}=0
$$

along the solutions of the differential equation $\left(G\left(t, x, q, q_{t}, q_{x}, \ldots\right)=0\right)$.

Moreover, if there exists a nontrivial differential function $Q$, called a 'multiplier', such that

$$
E_{q}[Q G]=0,
$$

then $Q G)$ is a total divergence, i.e.,

$$
Q G=D_{t} T^{t}+D_{x} T^{x},
$$

for some (conserved) vector $\left(T^{t}, T^{x}\right)$ and $E_{q}$ is the respective Euler-Lagrange operator. Thus, a knowledge of each multiplier $Q$ leads to a conserved vector determined by, inter alia, a Homotopy operator. See details and references in $[10,12]$. Below, we list the nontrivial conserved densities $T_{i}^{t}$ corresponding to the multipliers $Q^{i}$. It turns out that there are no derivative dependent multipliers.

(i) $Q^{1}=1$,

$$
T_{1}^{t}=q_{t}+\frac{1}{2} b_{2} q_{x x t}
$$

(ii) $Q^{2}=x$,

$$
T_{2}^{t}=x q_{t}+\frac{1}{6} b_{2}\left(-2 q_{x t}+3 x q_{x x t}\right)
$$

(iii) $Q^{3}=t$,

$$
T_{3}^{t}=-q+t q_{t}-\frac{1}{6} b_{2}\left(q_{x x}-3 t q_{x x t}\right)
$$

(iv) $Q^{4}=x t$,

$$
T_{4}^{t}=\frac{1}{6}\left(-6 x q+6 t x q_{t}+b_{2}\left(2 q_{x}-2 t q_{x t}-x q_{x x}+3 t x q_{x x t}\right)\right)
$$

The corresponding conserved quantities are therefore given by

$$
\begin{aligned}
I_{1} & =\int_{-\infty}^{\infty} T_{1}^{t} d x=\int_{-\infty}^{\infty}\left(q_{t}+\frac{b_{2}}{2} q_{x x t}\right) d x=0 \\
I_{2} & =\int_{-\infty}^{\infty} T_{2}^{t} d x=\int_{-\infty}^{\infty}\left(x q_{t}-\frac{b_{2}}{3} q_{x t}+\frac{b_{2}}{2} x q_{x x t}\right) d x \\
& =\frac{v A}{B} \frac{\Gamma\left(\frac{1}{2}\right) \Gamma\left(\frac{1}{2 n-1}\right)}{\Gamma\left(\frac{1}{2}+\frac{1}{2 n-1}\right)} \\
I_{3} & =\int_{-\infty}^{\infty} T_{3}^{t} d x=\int_{-\infty}^{\infty}\left(-q+t q_{t}-\frac{b_{2}}{6} q_{x x}+\frac{b_{2}}{2} t q_{x x t}\right) d x \\
& =-\frac{A}{B} \frac{\Gamma\left(\frac{1}{2}\right) \Gamma\left(\frac{1}{2 n-1}\right)}{\Gamma\left(\frac{1}{2}+\frac{1}{2 n-1}\right)}
\end{aligned}
$$




$$
\begin{aligned}
I_{4} & =\int_{-\infty}^{\infty} T_{3}^{t} d x=\int_{-\infty}^{\infty} \omega d x \\
& =\frac{t v A}{B} \frac{\Gamma\left(\frac{1}{2}\right) \Gamma\left(\frac{1}{2 n-1}\right)}{\Gamma\left(\frac{1}{2}+\frac{1}{2 n-1}\right)}=0
\end{aligned}
$$

where

$\omega=-x q+x t q_{t}-\frac{b_{2}}{3} q_{x}-\frac{b_{2}}{3} t q_{x t}-\frac{b_{2}}{6} x q_{x x}+\frac{b_{2}}{2} x t q_{x x t}$. The fourth conserved quantity is zero since this conservation law is valid at $v=0$, namely when the soliton is stationary.

\section{BIFURCATION ANALYSIS}

This section will carry out the bifurcation analysis of the Boussinesq equation with power law nonlinearity. Initially, the phase portraits will be obtained and the corresponding qualitative analysis will be discussed. Several interesting properties of the solution structure will be obtained based on the parameter regimes. Subsequently, the traveling wave solutions will be discussed from the bifurcation analysis.

\subsection{BIFURCATION PHASE PORTRAITS AND QUALITATIVE ANALYSIS}

To relate conveniently, let

$\alpha=\frac{a}{b_{1}+b_{2} v^{2}}$,

and

$\beta=\frac{k^{2}-v^{2}}{b_{1}+b_{2} v^{2}}$.

Setting $g^{\prime}=y$, then via (5), (50) and (51) we get the following planar system

$\left\{\begin{array}{l}\frac{\mathrm{d} g}{\mathrm{~d} \xi}=y, \\ \frac{\mathrm{d} y}{\mathrm{~d} \xi}=-\alpha g^{2 n}+\beta g .\end{array}\right.$

Obviously, the above system (52) is a Hamiltonian system with Hamiltonian function

$H(g, y)=y^{2}+\frac{2 \alpha}{2 n+1} g^{2 n+1}-\beta g^{2}$.

In order to investigate the phase portrait of (52), set

$f(g)=-\alpha g^{2 n}+\beta g$.

Obviously, $f(g)$ has two zero points, $g_{0}$ and $g_{*}$, which are given as follows

$g_{0}=0, \quad g_{*}=\left(\frac{\beta}{\alpha}\right)^{\frac{1}{2 n-1}}$.

Letting $\left(g_{i}, 0\right)$ be one of the singular points of system (52), then the characteristic values of the linearized system of system $(52)$ at the singular points $\left(g_{i}, 0\right)$ are

$\lambda_{ \pm}= \pm \sqrt{f^{\prime}\left(g_{i}\right)}$.
From the qualitative theory of dynamical systems, we know that

(i) If $f^{\prime}\left(g_{i}\right)>0,\left(g_{i}, 0\right)$ is a saddle point.

(ii) If $f^{\prime}\left(g_{i}\right)<0,\left(g_{i}, 0\right)$ is a center point.

(iii) If $f^{\prime}\left(g_{i}\right)=0,\left(g_{i}, 0\right)$ is a degenerate saddle point.

Therefore, we obtain the bifurcation phase portraits of system (52) in Fig.3.

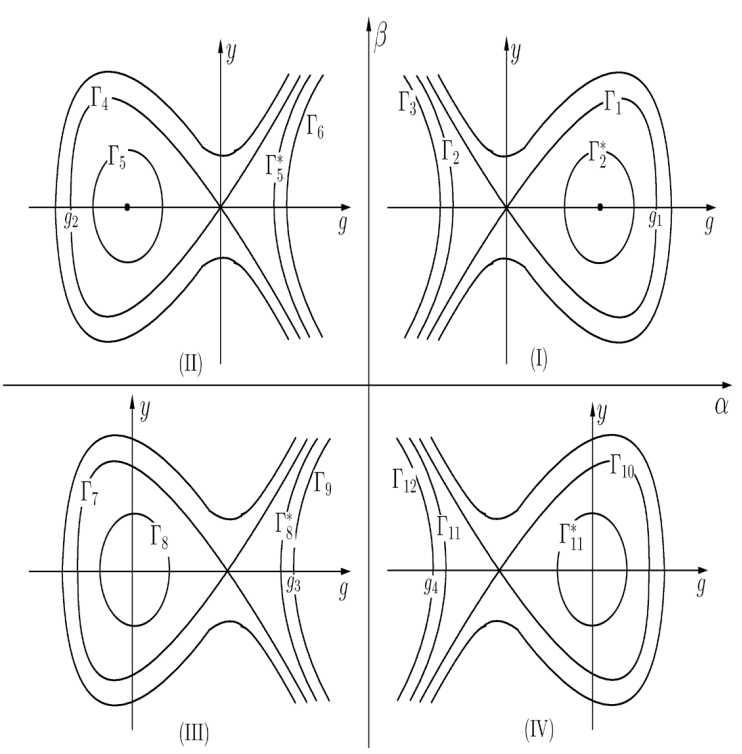

Fig. 3. The bifurcation phase portraits of system (52), (I) $\alpha>0$, $\beta>0$, (II) $\alpha<0, \beta>0$, (III) $\alpha<0, \beta<0$, (IV) $\alpha>0, \beta<0$

Let

$H(g, y)=h$,

where $h$ is Hamiltonian.

Next, we consider the relations between the orbits of (52) and the Hamiltonian $h$.

Set

$h^{*}=\left|H\left(g_{*}, 0\right)\right|$.

Proposition 1. When $\alpha>0$ and $\beta>0$, we have

(i) When $h=0$, system (52) has a homoclinic orbit $\Gamma_{1}$.

(ii) When $-h^{*}<h<0$, system (52) has two periodic orbits $\Gamma_{2}$ and $\Gamma_{2}^{*}$.

(iii) When $h=-h^{*}$, system (52) has a periodic orbit $\Gamma_{3}$.

(iv) When $h<-h^{*}$ or $h>0$, system (52) does not any closed orbit.

Proposition 2. When $\alpha<0$ and $\beta>0$, we have

(i) When $h=0$, system (52) has a homoclinic orbit $\Gamma_{4}$.

(ii) When $-h^{*}<h<0$, system (52) has two periodic orbits $\Gamma_{5}$ and $\Gamma_{5}^{*}$.

(iii) When $h=-h^{*}$, system (52) has a periodic orbit $\Gamma_{6}$. 
(iv) When $h<-h^{*}$ or $h>0$, system (52) does not any closed orbit.

Proposition 3. When $\alpha<0$ and $\beta<0$, we have

(i) When $h=h^{*}$, system (52) has a homoclinic orbit $\Gamma_{7}$.

(ii) When $0<h<h^{*}$, system (52) has two periodic orbits $\Gamma_{8}$ and $\Gamma_{8}^{*}$.

(iii) When $h=0$, system (52) has a periodic orbits $\Gamma_{9}$.

(iv) When $h<0$ or $h>h^{*}$, system (52) does not any closed orbit.

Proposition 4. When $\alpha>0$ and $\beta<0$, we have

(i) When $h=h^{*}$, system (52) has a homoclinic orbit $\Gamma_{10}$.

(ii) When $0<h<h^{*}$, system (52) has two periodic orbits $\Gamma_{11}$ and $\Gamma_{11}^{*}$.

(iii) When $h=0$, system (52) has a periodic orbits $\Gamma_{12}$.

(iv) When $h<0$ or $h>h^{*}$, system (52) does not any closed orbit.

From the qualitative theory of dynamical systems, we know that a smooth solitary wave solution of a partial differential system corresponds to a smooth homoclinic orbit of a traveling wave equation. A periodic orbit of a traveling wave equation corresponds to a periodic traveling wave solution of a partial differential system. According to above analysis, we have the following propositions.

Proposition 5. When $\alpha>0$ and $\beta>0$, we have

(i) When $h=0$, (1) has a solitary wave solution(corresponding to the homoclinic orbit $\Gamma_{1}$ in Fig.3).

(ii) When $-h^{*}<h<0$, (1) has a periodic wave solution and two periodic singular wave solutions(corresponding to the periodic orbits $\Gamma_{2}$ and $\Gamma_{2}^{*}$ in Fig.3).

(iii) When $h=-h^{*}$, (1) has two periodic singular wave solutions(corresponding to the periodic orbit $\Gamma_{3}$ in Fig.3).

Proposition 6. When $\alpha<0$ and $\beta>0$, we have

(i) When $h=0$, (1) has a solitary wave solution(corresponding to the homoclinic orbit $\Gamma_{4}$ in Fig.3).

(ii) When $-h^{*}<h<0$, (1) has a periodic wave solution and two periodic singular wave solutions(corresponding to the periodic orbits $\Gamma_{5}$ and $\Gamma_{5}^{*}$ in Fig.3).

(iii) When $h=-h^{*}$, (1) has two periodic singular wave solutions(corresponding to the periodic orbit $\Gamma_{6}$ in Fig.3).

Proposition 7. When $\alpha<0$ and $\beta<0$, we have

(i) When $h=h^{*}$, (1) has a solitary wave solution(corresponding to the homoclinic orbit $\Gamma_{7}$ in Fig.3).

(ii) When $0<h<h^{*}$, (1) has a periodic wave solution and two periodic singular wave solutions(corresponding to the periodic orbits $\Gamma_{8}$ and $\Gamma_{8}^{*}$ in Fig.3).

(iii) When $h=0$, (1) has two periodic singular wave solutions(corresponding to the periodic orbit $\Gamma_{9}$ in Fig.3).

Proposition 8. When $\alpha>0$ and $\beta<0$, we have

(i) When $h=h^{*}$, (1) has a solitary wave solution(corresponding to the homoclinic orbit $\Gamma_{10}$ in Fig.3). (ii) When $0<h<h^{*}$, (1) has a periodic wave solution and two periodic singular wave solutions(corresponding to the periodic orbits $\Gamma_{11}$ and $\Gamma_{11}^{*}$ in Fig.3).

(iii) When $h=0$, (1) has two periodic singular wave solutions(corresponding to the periodic orbit $\Gamma_{12}$ in Fig.3).

\subsection{TRAVELING WAVE SOLUTIONS}

Firstly, we will obtain the explicit expressions of traveling wave solutions for the (i) when $\alpha>0$ and $\beta>0$ (or $\alpha<0$ and $\beta>0$ ). From the phase portrait, we see that there is a homoclinic orbit $\Gamma_{1}$ (or $\left.\Gamma_{2}\right)$. In $(g, y)$-plane the expressions of the homoclinic orbit is given as

$y= \pm \sqrt{-\frac{2 \alpha}{2 n+1} g^{2 n+1}+\beta g^{2}}$.

Substituting (59) into $\frac{\mathrm{d} g}{\mathrm{~d} s}=y$ and integrating them along the orbits $\Gamma_{1}$ and $\Gamma_{2}$, we have

$\pm \int_{g_{1}}^{g} \frac{1}{\sqrt{-\frac{2 \alpha}{2 n+1} z^{2 n+1}+\beta z^{2}}} \mathrm{~d} z=\int_{0}^{s} \mathrm{~d} z$,
$\pm \int_{g_{2}}^{g} \frac{1}{\sqrt{-\frac{2 \alpha}{2 n+1} z^{2 n+1}+\beta z^{2}}} \mathrm{~d} z=\int_{0}^{s} \mathrm{~d} z$

Completing above integrals we obtain

$g= \pm\left(\frac{(2 n+1) \beta}{\alpha(1-\cosh ((2 n-1) \sqrt{\beta} s))}\right)^{\frac{1}{2 n-1}}$.

Using the notations of (2) and (3), we get the following singular solitary wave solutions

$q_{1 \pm}(x, t)= \pm\left(\frac{(2 n+1) \beta}{\alpha(1-\cosh ((2 n-1) \sqrt{\beta}(x-v t)))}\right)^{\frac{1}{2 n-1}}$,

where the parameters $\alpha$ and $\beta$ are given by (50) and (51) respectively.

Secondly, we will obtain the explicit expressions of traveling wave solutions for the (ii) when $\alpha<0$ and $\beta<0$ (or $\alpha>0$ and $\beta>0$ ). From the phase portrait, we note that there is a special orbit $\Gamma_{9}$ (or $\left.\Gamma_{12}\right)$. In $(g, y)$-plane the expressions of the orbit are given as

$y= \pm \sqrt{-\frac{2 \alpha}{2 n+1} g^{2 n+1}+\beta g^{2}}$.

Substituting (64) into $\frac{\mathrm{d} g}{\mathrm{~d} \xi}=y$ and integrating them along the two orbits $\Gamma_{9}$ and $\Gamma_{12}$, it follows that

$\pm \int_{g_{3}}^{g} \frac{1}{\sqrt{-\frac{2 \alpha}{2 n+1} z^{2 n+1}+\beta z^{2}}} \mathrm{~d} z=\int_{0}^{s} \mathrm{~d} z$

$\pm \int_{g_{4}}^{g} \frac{1}{\sqrt{-\frac{2 \alpha}{2 n+1} z^{2 n+1}+\beta z^{2}}} \mathrm{~d} z=\int_{0}^{s} \mathrm{~d} z$ 
$\pm \int_{g}^{\infty} \frac{1}{\sqrt{-\frac{2 \alpha}{2 n+1} s^{2 n+1}+\beta z^{2}}} \mathrm{~d} z=\int_{0}^{s} \mathrm{~d} z$

Completing above integrals we obtain

$g= \pm\left(\frac{(2 n+1) \beta}{2 \alpha} \sec ^{2}\left(\frac{(2 n-1) \sqrt{-\beta}}{2} s\right)\right)^{\frac{1}{2 n-1}}$

$g= \pm\left(\frac{(2 n+1) \beta}{2 \alpha} \csc ^{2}\left(\frac{(2 n-1) \sqrt{-\beta}}{2} s\right)\right)^{\frac{1}{2 n-1}}$.

From the notations of (2) and (3), we get the following periodic singular wave solutions

$q_{2 \pm}(x, t)= \pm\left(\mu \sec ^{2} v(x-v t)\right)^{\frac{1}{2 n-1}}$,

$q_{3 \pm}(x, t)= \pm\left(\mu \csc ^{2} v(x-v t)\right)^{\frac{1}{2 n-1}}$,

where $\mu=\frac{(2 n+1) \beta}{2 \alpha}, v=\frac{(2 n-1) \sqrt{-\beta}}{2}$, the parameters $\alpha$ and $\beta$ are given by (50) and (51) respectively.

\section{CONCLUSIONS}

This paper studied the BE with power law nonlinearity in presence of dual dispersion. There are three integration schemes that were applied to obtain soliton solutions. These are the traveling wave hypothesis, ansatz approach and finally the SVP. The ansatz method additionally produced shock waves and singular soliton solutions to BE. The wave profiles are also displayed numerically. The connection between singular solitons and solitary waves were also established. The conserved quantities were also obtained by the aid of multiplier method in Lie symmetry. Finally, the bifurcation analysis was carried out and phase portraits are also given. These results are going to be extremely useful in further future study of the equation in the context of shallow water waves.

The soliton perturbation theory will be established and addressed in future. The perturbation terms will also lead to the quasi-stationary soliton solution. Additional integration architectures will be employed in future to extract several other solutions. A few of them are the exp-function method, $G^{\prime} / G$-expansion method, simplest equation approach, Lie symmetry analysis and several others. These results will be reported in future.

\section{References}

[1] M. Alquran. Solitons and periodic solutions to nonlinear partial differential equation by the sine-cosine method. Applied Mathematics and Information Sciences, 6, 85-88 (2012).
[2] J. Angulo \& J. R. Quintero. Existence and orbital stability of cnoidal waves for a 1D Boussinesq equation. International Journal of Mathematics and Mathematical Sciences, 2007, 36 pages (2007).

[3] A. Biswas, D. Milovic \& A. Ranasinghe. Solitary waves of Boussinesq equation in a power law media. Communications in Nonlinear Science and Numerical Simulations, 44, 37383742 (2009).

[4] A. Biswas \& M. S. Ismail. 1-soliton solution of the coupled KdV equation and Gear-Grimshaw model. Applied Mathematics and Computation, 216, 3662-3670 (2012).

[5] A. Biswas, E. V. Krishnan, P. Suarez, A. H. Kara \& S. Kumar. Solitary waves and conservation law of Bona-Chen equation. Indian Journal of Physics, 87, 169-175 (2013).

[6] A. Biswas, D. Milovic, S. Kumar \& A. Yildirim. Perturbation of shallow water waves by semi-inverse variational principle. Indian Journal of Physics, 87, 567-569 (2013).

[7] A. G. Bratsos \& D. G. Natsis. A global extrapolated procedure for the Boussinesq equation". Journal of Applied Mathematics and Computing, 21, 23-43 (2008).

[8] G. Ebadi, S. Johnson, E. Zerrad \& A. Biswas. Solitons and other nonlinear waves for the perturbed Boussinesq equation with power law nonlinearity. Journal of King Saud University - Science, 24, 237-241 (2012).

[9] Awatif S. Jassim, Rasha A. Abdullah, Faleh L. Matar and Mohammed A. Razooqi. The Effect of Photo irradiation by Low Energy Laser on the Optical Properties of Amorphous GaAs Films. International Journal of Thin Films Science and Technology, 1, 9-13 (2012).

[10] U. Göktas and W. Hereman. Computation of Conservation Laws for Nonlinear Lattices. Physica D, 123, 425-436 (1998).

[11] A. J. M. Jawad, M. D. Petkovic, P. Laketa \& A. Biswas. Dynamics of shallow water waves with Boussinesq equation. Scientia Iranica: Transaction B., 20, 179-184 (2013).

[12] A. H. Kara. A symmetry invariance analysis of the multipliers and conservation laws of the Jaulent-Miodek and families of systems of KdV-type equations. Journal of Nonlinear Mathematical Physics, 16, 149-156 (2009).

[13] A. J. M. Jawad, M. D. Petkovic, P. Laketa \& A. Biswas. Dynamics of shallow water waves with Boussinesq equation. Scientia Iranica: Transaction B., 20, 179-184 (2013).

[14] Bo Zhang and Zhicai Juan, Modeling User Equilibrium and the Day-to-day Traffic Evolution based on Cumulative Prospect Theory. Information Science Letters, 2, 9-12 (2013).

[15] E. V. Krishnan, S. Kumar \& A. Biswas. Solitons and other nonlinear waves of the Boussinesq equation. Nonlinear Dynamics, 70, 1213-1271 (2012).

[16] Van Hieu Nguyen, Bich Ha Nguyen and Hai Trieu Duong. The quantum dynamics of two qubits inside two distant microcavities connected via a single-mode optical fiber. Advances in Natural Sciences: Nanoscience and Nanotechnology, 1, (2010).

[17] S. Noubissie, R. A. Kraenkel \& P. Waofo. Disturbance and repair of solitary waves in blood vessels with aneurysm. Communications in Nonlinear Science and Numerical Simulation, 14, 51-60 (2009).

[18] N. Polat \& E. Piskin. Existence and asymptotic behavior of solution of the Cauchy problem for the damped sixthorder Boussinesq equation. To appear in Acta Mathematicae Applicatae Sinica, English Series. 
[19] Kouji Tahata, Kouji Yamamoto and Sadao Tomizawa. Decomposition of Symmetry Using Palindromic Symmetry Model in a Two-Way Classification. Journal of Statistics Applications \& Probability, 1, 201-204 (2012).

[20] A-M. Wazwaz. New traveling wave solutions to the Boussinesq and the Klein-Gordon equations. Communications in Nonlinear Science and Numerical Simulation, 13, 889-901 (2008).

[21] J. B. Li, Z. R. Liu, Smooth and non-smooth traveling waves in a nonlinearly dispersive equation Appl. Math. Modell, 25, 41-56 (2000).

[22] K Mani Rahulan. S Ganesan and P Aruna. Synthesis and optical limiting studies of Au-doped $\mathrm{TiO} 2$ nanoparticles. Advances in Natural Sciences: Nanoscience and Nanotechnology, 2, (2012).

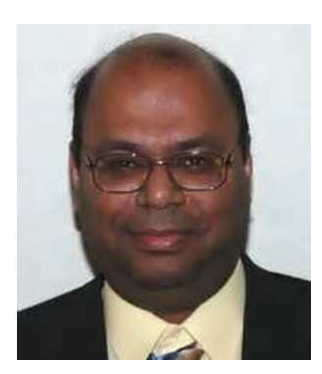

Anjan Biswas earned his B.Sc (honors) degree from Saint Xavier's College in Calcutta, India. Subsequently he received his M.Sc and M. Phil degrees in Applied Mathematics from University of Calcutta. Then, he earned MA and Ph.D. degrees in Applied Mathematics from the University of New Mexico in Albuquerque, New Mexico, USA. After that he carried out his Post-doctoral studies in Applied Mathematics from the University of Colorado, Boulder, USA. He was then an Assistant Professor of Mathematics at Tennessee State University that is located in Nashville, TN. Currently; he is working as an Associate Professor of Mathematics at Delaware State University in Dover, DE. His research interest is on Theory of Solitons.

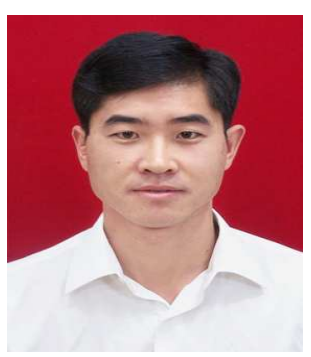

Ming

Song

is Professor of mathematics at Yuxi Normal University, China. $\mathrm{He}$ received the MS degree in applied mathematics from South China University of Technology. His research interests are in the areas of applied mathematics. He has published extensively in internationally refereed journals.

Houria Triki earned a

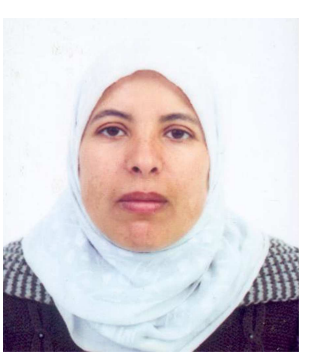
Ph.D. degree in Physics from Annaba University. Currently, she is a faculty member at Radiation Physics Laboratory at Badji Mokhtar University in Annaba, Algeria. She has published more than 60 papers in various peer-reviewed international journals of high repute and high impact factor. She has also participated in more than 60 scientific meetings.

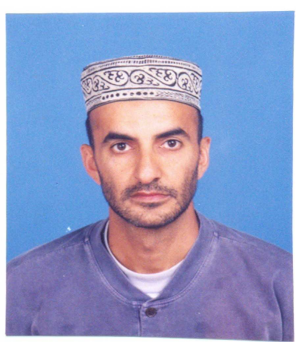

Abdul Hamid Kara is a professor in the School of Mathematics at the University of the Witwatersrand in Johannesburg, South Africa from where he obtained his $\mathrm{PhD}$. His field of expertise involves the analysis of differential equations using invariance properties and conservation laws. He has published, collaboratively, in the areas of mathematical physics, relativity and cosmology, applications of differential geometry, classification of Lagrangians, inter alia. Prof Kara has supervised a number of $\mathrm{MSc} / \mathrm{PhD}$ students and is an NRF rated researcher in South Africa.

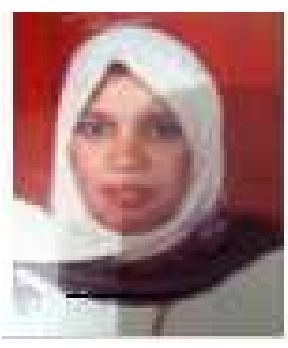

Bouthina S. Ahmed Doctor of Numerical Analysis in Mathematics Department - Women Science -Ain Shams University - Cairo - Egypt. Her MS degree in differential equations and Ph.D degree in numerical analysis. Her research interests in applied mathematics.

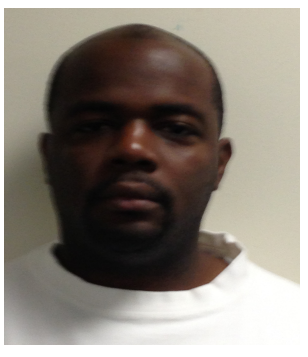

Andre Strong earned his BS in Mathematical Sciences from Tennessee State University in Nashville, TN. Subsequently, he received his MS Mathematical Sciences from Tennessee State University also. His MS thesis was entitled "Robust Stability and Stabilization of a Class of Non-Linear Discrete-Time Stochastic Systems". Currently he is pursuing his doctoral studies in Applied Mathematics at Delaware State University, Dover, DE, USA. His research area is "Theory of Solitons".

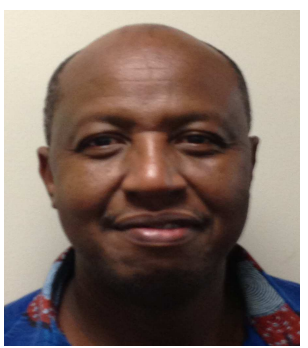

Amadou Hama earned his BS in Mathematics from University of Jos (Nigeria).Subsequently, he gained M.Sc degree in Mathematics from University of Jos followed by an MS from Louisiana State University, Baton Rouge, LA, USA. At present, he is pursuing doctoral studies in Applied Mathematics at Delaware State University in Dover, DE, USA. His research area of interest is 'Theory of Solitons". 